\title{
Innovation et reconversion territoriale : l'apport des réseaux dans le secteur du multimédia
}

\author{
Diane-Gabrielle Tremblay et Serge Rousseau \\ Télé-université de l'Université du Québec à Montréal
}

\section{Introduction}

$\mathcal{L}$ a région métropolitaine de Montréal connaît un dynamisme économique renouvelé. Passée d'une économie axée sur des activités manufacturières traditionnelles, elle a réussi son virage vers une économie orientée sur le savoir et l'innovation. Selon la firme de consultants Pricewaterhouse Coopers ${ }^{1}$, la région montréalaise se positionne au quatrième rang en Amérique du Nord pour ce qui est de la densité d'emplois per capita en haute technologie, derrière San Francisco (incluant la Silicon Valley), Seattle (avec les entreprises Boeing et Microsoft) et Boston.

Le secteur du multimédia a fortement participé à la reconversion industrielle et territoriale de l'économie montréalaise, contribuant ainsi à se sortir de la morosité de la crise des années 1980. Le secteur fait partie des secteurs de haute technologie qui caractérisent la réalité montréalaise et ce, au même titre que les secteurs de l'aéronautique, des télécommunications et de la biopharmaceutique ${ }^{2}$.

Le secteur du multimédia est relativement jeune. S'il apparait vers le début des années 1990 avec l'avènement de l'Internet, ce ne sera que vers le milieu de cette décennie que l'on pourra véritablement le désigner comme un secteur distinct et que l'on observera sa contribution particulière au développement de l'innovation ${ }^{3}$ et à la reconversion territoriale, principalement à Montréal mais aussi à Québec ${ }^{4}$.

Le multimédia a également eu d'autres effets. Au plan urbanistique, il a contribué à la revitalisation et à la requalification de nombreuses anciennes zones industrielles ou commerciales. Il suffit de penser au Faubourg des Récollets et au boulevard Saint-Laurent à Montréal ou encore au quartier Saint-Roch à Qué- bec. Ce phénomène n'est pas exclusif au Québec. Aux États-Unis, le quartier South Market à San Francisco ou encore la Silicon Alley à New York, ou encore le Sentier à Paris sont des exemples de réappropriation et de requalification urbaine similaire à ce qui se passe sur le territoire montréalais ${ }^{5}$. L'émergence du secteur a contribué aussi au retour des activités de haute technologie vers le noyau ancien de la ville. Il s'agit en quelque sorte du renversement d'une tendance lourde, car les entreprises œuvrant dans la haute technologie avaient délaissé la ville centrale au profit de zones périphériques. Les entreprises du secteur s'approprient ainsi de vieux immeubles industriels, aux espaces ouverts, favorisant ainsi la création d'un climat de travail plus convivial et des échanges fréquents entre travailleurs, comme le souhaitent nombre d'entrepreneurs du secteur du multimédia. Le secteur a donc un impact important sur le plan de la reconversion territoriale des centres-villes. Mais comment expliquer que le secteur du multimédia se soit établi dans les zones centres? La recherche de réseautage expliquerait le choix des zones urbaines centrales et, par le fait même, la reconversion de ces zones.

L'objet de cet article est de tenter de mieux comprendre le phénomène du réseautage, ses modalités comme ses obstacles, afin de jeter de la lumière sur le processus d'innovation et de reconversion des territoires qui en résulte, et dans ce cas-ci, au développement du multimédia. Les théories de l'innovation ont beaucoup évolué au fil des ans. Alors que les modèles initiaux de l'innovation étaient plutôt de nature linéaire, fondés sur la R\&D, les modèles plus récents traitaient de chaînes de liaisons, de réciprocité d'effets, puis finalement de systèmes (régionaux ou nationaux) d'innovation $^{6}$. La théorie des systèmes régionaux ou nationaux d'innovation, issue des thèses évolutionnistes, 
considère l'innovation comme un processus issu d'interactions entre les acteurs, un processus qui conduit à des apprentissages et n'est pas du tout linéaire, mais fondé sur des allers-retours, liaisons et boucles d'apprentissage ${ }^{7}$. Ceci conduit à accorder plus d'importance aux échanges entre acteurs, organismes et institutions dans un territoire donné. Dans les théories des milieux innovateurs, ou clusters, élaborées par la suite, on postule généralement que le réseautage et les liens interentreprises contribuent fortement au développement territorial et à l'innovation grâce aux échanges d'information et à l'apprentissage qu'ils permettent ${ }^{8}$.

En vue de comprendre ce processus, le présent article se divise en deux parties. La première partie présente une typologie des réseaux, alors que la seconde s'intéresse à la dynamique des entreprises et des réseaux que nous avons observés dans le secteur multimédia, se penchant sur les facteurs favorables et les obstacles au réseautage. En conclusion, nous tenterons de dégager de ce cas quelques leçons d'ordre plus général concernant le lien entre innovation et territoires.

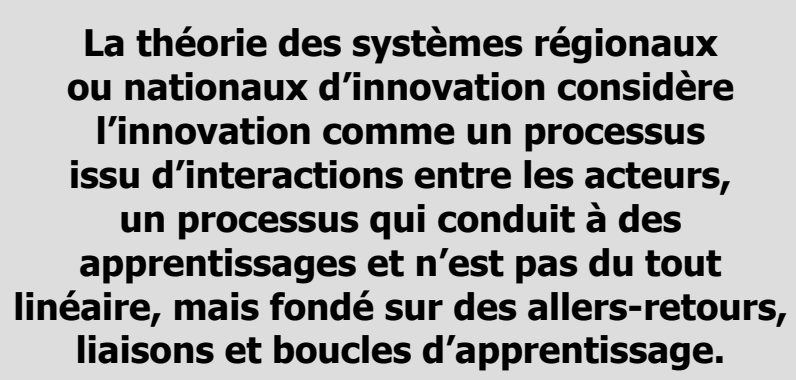

\section{Typologie des réseaux}

Il est possible d'établir une typologie des réseaux en trois catégories : le réseau vertical, le réseau horizontal et le réseau intermédiaire. Ces réseaux contribuent chacun à leur manière à la dynamique territoriale. Ils renvoient à l'ensemble des acteurs qui sont susceptibles d'avoir une incidence sur le développement du secteur et du territoire.

\section{Le réseau vertical}

Le réseau vertical renvoie à l'existence de relations entre des unités productives pour la réalisation d'un bien ou d'un service, des relations autres que strictement marchandes de type vendeur-acheteur; il suppo- se des relations de coopération et de partenariat qui peuvent se concrétiser à l'intérieur de la sphère marchande, mais également à l'extérieur de celle-ci. Il est constitué strictement d'entreprises. Emanuel ${ }^{9}$ propose une typologie de réseau d'entreprises :

«- Les districts technologiques : interaction prédominante entre les activités de direction, de R\&D, de services spécialisés externes, technologiques plus élevés que dans les entreprises manufacturières.

- Les réseaux situés autour de grands établissements à caractère local.

- Présence de sièges sociaux qui maintiennent des liens avec le territoire d'où ils proviennent et des unités de production qui appartiennent non seulement au secteur manufacturier mais aussi aux services spécialisés et aux services de support à la production et à la commercialisation.

- Les réseaux des zones-systèmes et des districts industriels: présence importante de PME œuvrant dans le même secteur.

- Les réseaux de systèmes de productions locaux : présence importante de PME spécialisées dans le même secteur avec une production qui peut être similaire.

- Les réseaux de productions spécialisées : présence importante de PME ayant une structure horizontale, qui produisent toutes le même bien ou effectuent la même phase de travail et sont donc concurrentes sur le marché.

- Les réseaux résultant d'une "verticalisation" des grandes firmes ${ }^{10}$ : leur formation dérive de stratégies de réorganisation des grandes entreprises. »

\section{Le réseau horizontal}

Le réseau horizontal fait référence à des acteurs qui œuvrent à l'intérieur des espaces de solidarité, capables d'engendrer des ressources et des compétences nouvelles dont peuvent bénéficier l'ensemble des entreprises du secteur. Ce type de réseau cherche à assurer la pérennité et le développement du secteur comme du territoire. Contrairement au réseau vertical, le réseau horizontal est beaucoup plus diversifié. Outre les unités productives, il peut être composé de différentes associations à vocation sectorielle ou spatiale, des organismes de tous types (publics, privés et parapublics), des administrations publiques (fédéral, pro- 
vincial, régional, municipal et local), des établissements d'enseignement, des individus, etc. De plus, ce n'est pas l'entreprise qui est directement concernée ici mais plutôt le secteur ou l'espace de référence des agents impliqués.

\section{Le réseau intermédiaire}

Le réseau intermédiaire renvoie aux relations qui interpellent, d'une part, les entreprises et, d'autre part, les organismes qui peuvent soutenir les activités de celles-ci. La finalité de ces organismes ne réside pas dans la réalisation et la production de biens et services, mais s'oriente plutôt vers une démarche d'accompagnement dans l'accomplissement de la finalité de l'entreprise. Le réseau intermédiaire peut inclure plusieurs types d'acteurs. Outre les entreprises, on retrouve les institutions d'enseignement, les centres de recherches, les incubateurs, les entreprises de financement (institutions financières et société de capital de risque), les sociétés de conseil (comptable, ingénieurs, avocats...), les entreprises de distribution.

Dans le cadre de notre recherche, nous avons tenté de voir si le secteur du multimédia était caractérisé par certaines formes de réseautage, et dans quelle mesure ces activités ont pu contribuer au développement territorial et au développement de l'innovation.

\section{Le réseautage dans le secteur du multimédia}

Il faut d'abord rappeler que le secteur du multimédia est un secteur jeune et que la réalisation du produit final est relativement simple, comparativement à la construction d'un avion ou d'une automobile, par exemple. De ce fait, la chaîne de production est relativement simple et peut limiter les occasions de réseautage. Cependant, la recherche d'innovation est importante dans ce secteur et, sous cet angle, la recherche et l'élaboration du produit innovant sont souvent d'une complexité technologique importante. Ainsi, nous avons observé que le réseautage entre entreprises était moins important qu'on aurait pu le penser, mais qu'il commence à se développer et que d'autres formes de réseautage (horizontal et intermédiaire) ont aussi un certain rôle à jouer dans le développement territorial et l'innovation.

Avant d'entrer dans la présentation des résultats de recherche, quelques éléments sur la méthodologie.
Précisons d'abord que nous avons réalisé une enquête en 2003 et 2004 auprès de 75 intervenants qui œuvrent dans les différentes sphères du multimédia. Outre les entreprises, nous avons rencontré les sociétés de capital de risque, les organismes de développement économique, les différentes administrations publiques ainsi que des consultants afin d'obtenir les points de vue de tous les acteurs. Ceci nous a permis de comprendre les particularités du secteur, les pratiques de réseautage et les stratégies des acteurs publics et privés.

Une des particularités du multimédia, c'est que la complexité du produit réside dans la conception du produit et non dans le produit final comme tel. Le produit final se limite souvent à un Cédérom, soit un produit assez simple à fabriquer. C'est dans la recherche d'innovations et la conception ou le design du produit que se trouve toute la complexité. La majorité des produits se réalise à l'intérieur d'une organisation, avec peu d'échanges à l'externe. Ceci est particulièrement vrai lorsque la taille de l'entreprise est petite.

De plus, certaines entreprises préfèrent éviter d'externaliser une partie de la production, car elles y voient une perte de revenu. Selon certains interlocuteurs, la conjoncture difficile du secteur rend difficile le recours à des partenaires, alors que ceci pouvait se faire davantage auparavant. "[...] pardonnez le français, mais on est "self sustained " [...] On a nos ressources, nos "games designers", nos designers visuels, nos «writers » internes, nos programmeurs internes, nos ingénieurs internes, nos chargés de projet, tout, tout, tout $[\ldots]$ tout est à l'interne. » $(\mathrm{S})$

\section{Une des particularités du multimédia, c'est que la complexité du produit réside dans la conception du produit et non dans le produit final comme tel.}

Il n'est donc pas rare de voir des entreprises concevoir leur produit tout à fait seules : « Ça a été conçu [le produit] de $\mathrm{A}$ à $\mathrm{Z}$ à l'intérieur de $\mathrm{D}$. » (D)

On retrouve la même chose dans la création de type animation : " On n'a aucune association, aucun partenariat avec des entreprises. On a des partenariats avec des centres universitaires pour l'accréditation de programmes de formation médicale continue, on a un partenariat avec l'université McGill, mais on n'a pas 
de partenariat ou d'ententes commerciales avec aucune entreprise québécois ou canadienne. » (C) « Nous, on a une filiale, on a deux compagnies, il y a une compagnie mère qui est $\mathrm{K}$. Pis on a une filiale qui fait la production, $\mathrm{K}$ étant la maison de services dans l'animation. » $(\mathrm{K})$

L'ensemble de nos entretiens nous portent donc à conclure qu'il n'est pas facile de développer des activités de réseautage vertical entre les entreprises, surtout pour les plus petites. En effet, certaines des plus grandes vont parfois faire faire des choses à l'extérieur, mais c'est aussi parfois limité, car on souhaite conserver les compétences et les connaissances relatives à l'innovation à l'interne. Nous verrons d'abord quels motifs alimentent la formation de réseaux dans le secteur du multimédia, avant de passer à l'identification des obstacles au développement du réseautage, une dimension importante de la recherche puisqu'elle fournit des éléments de solution ou de recommandation pour le développement du réseautage en vue d'accroître le dynamisme innovateur et le développement de certains territoires.

\section{Les facteurs favorables au développement des réseaux}

De façon générale, le développement de réseaux exige du temps. On nous a indiqué que cette dimension temporelle est importante pour trois raisons : d'une part, pour établir une notoriété de l'entreprise par rapport à son environnement; d'autre part, afin de connaître les entreprises qui sont susceptibles de développer des relations résiliaires; et finalement, pour établir des relations de confiance entre les individus. La notoriété et la confiance sont des concepts à double dimensions, c'est-à-dire que l'entreprise doit avoir à l'égard de son partenaire un certain niveau de confiance, mais elle doit aussi susciter elle-même un climat de confiance afin d'être perçue comme un partenaire convenable pour l'autre partie. La relation de confiance ne s'élabore pas seulement en termes de complémentarité des capacités productives des entreprises, même dans un secteur de haute technologie; nous avons constaté qu'elle repose souvent sur la complicité entre les individus associés à un projet, sur le respect établi au fil du temps. «Tu ne peux pas te débattre contre un gros joueur, et il faut juste que tu trouves une façon intelligente d'amener une stratégie pour que ces gens-là te respectent. Et je pense qu'un des plus gros signe de respect, c'est avec les années.
On a dix ans maintenant, et on a beaucoup plus de respect maintenant qu'on avait il y a 10 ans, y'a 5 ans, parce que les gens te reconnaissent comme une compagnie établie. Que tu sois plus petite, c'est une chose, mais vu que t'as survécu à des époques, les gens vont avoir un respect beaucoup plus profond au niveau de l'organisation, ils vont être beaucoup plus réticents à vouloir être chiens avec toi puisque bon... y'a un respect qui s'est installé. » (K)

\section{La relation de confiance ne s'élabore pas seulement en termes de complémentarité des capacités productives des entreprises.}

Malgré tout, il est possible de voir des associations se développer avec d'autres entreprises et les entreprises intermédiaires jouent parfois un rôle dans le développement de tels réseaux. «Oui, on est associé avec une entreprise de publicité et de communication. En fait, on fait partie d'un groupe qui s'est associé différents métiers de la communication. Et on développe des affaires avec ce groupe-là. » (I) «[...] moi ici, je travaille comme un producteur de cinéma. Ça veut dire que c'est pas plus gros que ça. J'ai un infographe en chef, j'ai mon adjointe, y'a un directeur technique. On est 4 . Des fois 5. Ça dépasse pas ça. Mais y'a des saisons où on peut être 18 , tout dépendant des mandats que je prends. Je donne beaucoup de contrats à l'extérieur. Toute ma programmation en $\mathrm{X}$, c'est L qui la fait. Ils ont leur atelier, ils sont incorporés. Donc régulièrement, $\mathrm{T}$ ça peut être $12,14,16$ personnes qui travaillent, qui pivotent autour. Mais le cœur, c'est 4 personnes. » $(\mathrm{T})$

Les collaborations peuvent aussi s'établir sur le plan technique afin d'accéder à des équipements qu'il pourraient être trop onéreux d'acquérir. «Oui, y’a C qui s'occupe de nos serveurs [...] tu vois, et ça c'est important. Tout le côté hosting est hébergé chez eux. Que je ne dise pas de bêtises, le reste [...] On a travaillé avec les représentants locaux de B, le côté équipements, le côté réseau [...] ça on l'a fait avec des gens d'ici. » « Donc, j'ai développé à titre personnel certaines expertises ou connaissances en termes de gestion de programmes pour répondre aux besoins, ou en fait aux critères ici des programmes, mais c'est pas nécessairement ce dans quoi je suis meilleur. Donc, c'est peut-être d'utiliser les ressources au meilleur escient, effectivement. »(Y) 
Les collaborations peuvent aussi avoir pour but de simplifier la distribution: "Pour les firmes avec lesquelles on est partenaires dans des alliances stratégiques, on a Broad Education aux États-Unis qui lui s'occupe de tout ce qui est la diffusion en ligne. $\mathrm{Au}$ Canada, on a des ententes de partenariat et de production avec la Sofad, Samfo en Ontario, Alberta Learning en Alberta, Thomson un éditeur, Thomson Learning, puis on des petits partenariats à gauche et à droite. On a notre distributeur en France pour la francophonie. Y'a des alliances stratégiques comme ça. » (C)

\section{Les obstacles à la formation de réseaux verticaux dans le secteur du multimédia sont nombreux.}

Les alliances ou le réseautage peuvent aussi porter sur le contenu : «Donc, il faut pour le moins réaliser [...] faire preuve d'imagination et réaliser des partenariats qui nous donnent accès [...] en tout cas, moi, qui me donnent accès à d'autres contenus que justement je ne peux pas m'alimenter auprès d'Art Global, selon les types de produits que je développe. Une partie vient d'Art Global, il m'en manque une autre partie, donc je vais la chercher dans des musées ou ailleurs. Le produit que je réalise actuellement, qui est vraiment un projet d'envergure, là je me suis associée à une firme audio-visuelle dont le savoir-faire était plutôt axé vers la télévision, moi vers le multimédia, ce qui nous a permis de réaliser un produit convergent qui rentre aussi dans les critères d'admissibilité de plusieurs fonds qui veulent justement ce type de produitlà. Alors il faut faire preuve d'imagination si on veut survivre. » (E) « À Toronto, y'a beaucoup d'affiliations avec des fournisseurs de contenus externes. Y'en a des centaines. Donc, souvent c'est des deals [...] on échange les contenus en échange de visibilité, nous ça nous fournit du contenu. Donc, par exemple, je sais pas, on peut faire un deal avec la NFL [...] à Toronto on a un site, le site canadien de la NFL est géré par Canoe à Toronto. Donc, les contenus viennent de la NFL, nous ça nous fournit des contenus, puis on a un échange de [...] si on met de la publicité là-dedans, y'a un modèle d'affaires où on "split" les locaux. » $(\mathrm{N})$ « Comment est-ce que ça fonctionne ici, c'est que $\mathrm{T}$, nos partenaires qui ont des contrats [...] disons que la plupart des contrats qu'on fait ici c'est des logiciels pour Loto-Québec, pour les loteries télé- visées, ou des jeux à la télé. [...] Alors quand eux ont des contrats, ils viennent ici, puis on s'occupe de faire les logiciels. » (X)

Les associations peuvent souvent être temporaires et ponctuelles étant donné la particularité du secteur du multimédia qui repose largement sur une production par projet. «Par contre, il peut arriver à l'occasion qu'on puisse octroyer des contrats de sous-traitance ou des choses comme ça quand la ressource qu'on a ici sur place ne nous permet [...] si on a un manque de ressources, c'est déjà arrivé à l'occasion. » (U)

Il y a aussi des partenariats qui sont en voie de se constituer: "On veut [...] le genre de partenariat qu'on est en train de faire, on veut que ce soit ça, c'est-à-dire que chacun a sa spécialité, puis ensemble on peut offrir une gamme, une solution plus complète et plus avant-gardiste que seulement un ou deux autres. Fait que qu'est-ce qu'on est en train de faire, oui, c'est ça $[\ldots]$ puis je pense qu'y a une certaine ouverture aussi à $[\ldots]$ par d'autres firmes, de ne pas être nécessairement bons en tout, et de se faire des partenariats, puis chacun son plus [+] là-dedans apporte [...] et permet de développer des affaires qu'elles auraient jamais pu faire. » (Y) « On a des associations avec certaines firmes de communication parce que c'est un outil qu'ils peuvent offrir à leurs clients. On a également une association avec une entreprise de Sherbrooke qui fait de l'hébergement de sites Web parce qu'on offre quand même une partie hébergement, archivage des messages, et tout ça [...] qu'on travaille avec eux. Donc, on a quelques autres partenaires $[. .$.$] avec par exemple, on est en train de déve-$ lopper un partenariat avec une entreprise en Europe qui va commercialiser notre produit en Europe. » (L)

Les extraits d'entrevue témoignent bien du fait que le réseautage n'est pas encore une pratique courante dans le multimédia, mais qu'il se développe de plus en plus. On note également que le réseautage ne concerne pas seulement des entreprises, mais parfois aussi des organismes intermédiaires ou offrant d'autres services (distribution, etc.). Les réseaux horizontaux et intermédiaires se développent donc aussi, venant compléter parfois les réseaux verticaux, ou partenariats entre entreprises, qui sont parfois plus ponctuels et temporaires mais visent à obtenir l'accès à des équipements, à une distribution ou à des contenus. Nos entretiens ont aussi permis de mettre en évidence les éléments qui peuvent nuire au développement des 
réseaux, et c'est à ces obstacles que nous nous intéressons maintenant.

\section{Les obstacles au développement des réseaux}

Les obstacles à la formation de réseaux verticaux dans le secteur du multimédia sont nombreux. Les contraintes et les limites sont nombreuses pour expliquer le plus faible niveau d'activités résiliaires comparativement à d'autres secteurs.

\section{La dynamique interne du secteur}

La dynamique interne du secteur est un facteur qui réduit le potentiel du développement du réseau. Les nombreuses transformations, fusions liées à la convergence des activités, faillites et autres éléments contribuant au développement de la concurrence ont contribué à créer un climat d'incertitude, nuisant à la coopération. " La difficulté est l'instabilité. On établit de bons liens, on développe des produits que nos clients aiment, et du jour au lendemain, un changement chez le partenaire nous amène à repenser notre stratégie. » (A)

\section{La jeunesse des entreprises}

Dans un secteur jeune comme le multimédia, bien qu'il puisse y avoir entrée de joueurs d'autres secteurs (comme l'édition ou d'autres médias), la grande majorité des entreprises sont très jeunes, un grand nombre ayant moins d'une dizaine d'années. Notre enquête montre que les entrepreneurs en sont souvent à leur première expérience dans le monde des affaires. Les efforts lors du démarrage de l'entreprise sont souvent concentrés sur des activités internes et le développement du réseautage ne vient que plus tard. "Et làdessus, y'en avait plus de $50 \%$ qui avait peut-être, j'ai pu les chiffres, mais cinq employés et moins, là on tombait dans les très petites entreprises. Et quand t'es petit comme ça, tu portes à bout de bras, t'es le producteur, président, promoteur, tu fais tout [...] donc il faut vraiment que t'aies une occasion [...]» (V) Ou encore : «[...] mais je dirais que dans les compagnies qui ont un peu plus de maturité, qui ont quelques années en affaires, là il se développe des relations [...] Parce que c'est sûr que ça ne se crée pas à partir de zéro, il faut une certaine dose de confiance pour pouvoir se mettre ensemble, pour faire des consortiums, des regroupements [...] Et puis les entreprises qui démarrent ont d'autres chats à fouetter. » (V)

\section{La durée de vie de produit}

La réduction du temps de développement des produits ainsi que le respect des dates de lancement sont des facteurs décisifs de succès pour les entreprises. L'industrie du multimédia bouge rapidement et est souvent impitoyable. La réalisation d'un produit connaissant un succès n'est pas garante de la pérennité de l'entreprise, même à court terme. Dans l'industrie du jeu, la durée de vie est évaluée, pour un jeu ayant un certain succès, à 18 mois, soit approximativement la même durée que son développement. Dans le logiciel, les «mises à jour » doivent être continuelles, c'est-àdire au moins annuellement, pour conserver les parts du marché acquis.

Selon nos interlocuteurs, un environnement en perpétuelle mouvance et mutation, avec une durée de vie relativement courte des produits, rend difficile le développement de réseaux entre entreprises, mais peut parfois mener à rechercher des liens plus étroits avec d'autres types de partenaires (sources de capital de risque, banque, organisme public de soutien, etc.), contribuant ainsi au développement de réseaux horizontaux ou intermédiaires.

\section{La faible production locale}

Dans le cas de la production multimédia à Montréal ou au Québec, il faut également prendre en considération le faible volume de production locale du secteur. « Mais il faut agrandir, parce que le marché local de la sous-traitance, dans un domaine hyper spécialisé comme le son, l'image 3D, ou l'ingénierie linguistique comme moi $[\ldots]$ ben ça déborde [...] la demande n'est pas assez forte localement, il faut donc avoir des clients internationaux et ça, c'est pas évident non plus à développer. Fait que c'est pour ça que la soustraitance est peut-être un peu déficiente. » (L)

Le faible volume fait en sorte que la production est relativement atomisée, c'est-à-dire qu'elle est répartie entre les différents sous-secteurs. Le faible volume fait qu'il est parfois difficile pour une entreprise d'exploiter un seul créneau précis, ou une expertise donnée, car le marché québécois serait insuffisant pour rentabiliser une telle orientation. On cherche alors des clients à l'échelle internationale, mais jusqu'ici, peu d'entreprises ont développé des réseaux à cette échelle, généralement parce qu'elles ont peu de moyens ou de personnel à y consacrer. 


\section{Conclusion}

Notre recherche a montré qu'il n'est pas facile de développer des activités de réseautage vertical entre les entreprises du multimédia, surtout pour les plus petites. Nous avons malgré tout observé l'émergence de réseaux, principalement des réseaux s'apparentant à des réseaux de PME à production spécialisée, mais aussi un peu de verticalisation issue des grandes entreprises. Au nombre des motifs alimentant la formation de réseaux, nous avons observé la recherche de complémentarités de production, l'accès à des équipements, à des contenus ou à des réseaux de distribution.

En ce qui concerne les obstacles au développement de réseaux, la durée de vie du produit et la rapidité des changements requis dans le secteur sont incontournables. Par contre, le secteur commence à prendre de la maturité et les jeunes entrepreneurs ont pris de l'âge, se sont développé des connaissances, voire des réseaux. Des organismes intermédiaires pourraient aussi favoriser ce développement, comme l'ont fait certaines associations comme l'Association des producteurs multimédia ou d'autres. La dynamique interne du secteur s'est un peu stabilisée et la production locale pourrait possiblement être favorisée par le développement de réseaux entre entreprises de diverses régions du Québec.

On note également que le réseautage ne concerne pas seulement des entreprises, mais parfois aussi des organismes intermédiaires ou offrant d'autres services (distribution, etc.). Ceux-ci viennent occasionnellement compléter les réseaux verticaux, ou partenariats entre entreprises, qui sont parfois plus ponctuels et temporaires, mais visent à obtenir l'accès à des équipements, à une distribution ou à des contenus.

\section{En ce qui concerne les obstacles au développement de réseaux, la durée de vie du produit et la rapidité des changements requis dans le secteur sont incontournables.}

Notre recherche a donc permis de mieux comprendre le phénomène du réseautage, ses modalités comme ses obstacles et, de ce fait, de mieux comprendre également le processus d'innovation et de reconversion des territoires grâce à l'innovation. Comme nous l'avons indiqué en introduction, les théories de l'innovation ont évolué au fil des ans, mais les théories linéaires plus anciennes, fondées sur la $R \& D$, ne sont pas totalement disparues du paysage. Notre recherche a toutefois permis d'illustrer le fait que, dans le cas du multimédia, ce sont les théories plus récentes qui s'appliquent, car l'innovation dans ce secteur ne repose pas tant sur la $R \& D$ classique, en laboratoire, que sur des échanges entre acteurs, des apprentissage issus du réseautage. La théorie des systèmes régionaux ou nationaux d'innovation, issue des thèses évolutionnistes, se trouve ici illustrée puisqu'il s'agit bien d'un processus issu d'interactions entre les acteurs, un processus qui conduit à des apprentissages et n'est pas du tout linéaire, mais fondé sur des allers-retours, liaisons et boucles d'apprentissage. Les échanges entre acteurs, organismes et institutions dans un territoire donné sont déterminants pour le processus d'innovation. Comme le veut la théorie des milieux innovateurs ou des clusters, le réseautage et les liens interentreprises contribuent fortement au développement territorial et à l'innovation grâce aux échanges d'informations et à l'apprentissage qu'ils permettent ${ }^{11}$.

Sur le plan des politiques publiques visant à accroître le dynamisme innovateur et le développement de certains territoires, ceci signifie qu'il ne faut pas se limiter au soutien à la R\&D, mais élargir les modes d'appui à l'innovation. Ainsi, on pourrait imaginer que les zones qui cherchent à favoriser une reconversion territoriale pourraient avoir intérêt à mettre en place des organismes intermédiaires qui fourniraient de tels services aux entreprises, par exemple la mise en commun d'équipements, le partage de certains contenus, l'offre de distribution au Québec et surtout à l'étranger, autant de services qui permettent de développer les échanges d'informations et l'apprentissage entre les acteurs.

\section{Notes et références}

1 PricewaterhouseCoopers (1999). 1998 British Columbia New Media Industry Survey.

2 Selon Montréal International (2002), la région montréalaise se situerait au $9^{\mathrm{e}}$ rang dans le secteur des technologies de l'information, au $4^{\mathrm{e}}$ rang dans l'aérospatiale et au $8^{\mathrm{e}}$ rang pour le biopharmaceutique au niveau des métropoles nord-américaines.

3 Tremblay, D.-G. et S. Rousseau (2005a). « The Montreal Multimedia Sector : A Cluster, an "Innovative Milieu" or a 
Simple Colocation?», Canadian Journal of Regional Science, accepté pour publication, à paraître en 2006.

4 Manzagol, C., É. Robitaille, et P. Roy. (2000). « Le multimédia à Montréal : le high-tech à la rescousse des espaces fatigués ", dans G. Sénécal et D. Saint-Laurent, Les espaces dégradés. Contraintes et conquêtes, Montréal, Université de Montréal; Tremblay, D.-G. et S. Rousseau (2005). "Le secteur du multimédia à Montréal peut-il être considéré comme un milieu innovateur? », Géographie, Économie et Société, Paris, Lavoisier, p. 37-56; Tremblay, D.-G., C. Chevrier et S. Rousseau (2004). « The Montreal Multimedia Cluster : District, Cluster or Localized System of Production? », dans D. Wolfe and M. Lucas (dir.), Clusters in a Cold Climate : Innovation Dynamics in a Diverse Economy, Montreal and Kingston, McGill-Queen's University Press and School of Policy Studies, Queen's University, p. 165-194.

5 Manzagol, C. et G. Sénécal (2002). «Introduction. Les grands projets et le destin métropolitain », dans G. Sénécal, J. Malézieux et C. Manzagol (dir.), Grands projets urbains et requalification, Québec, Presses de l'Université du Québec.

6 Tremblay, D.-G. (1995). «La multidimensionnalité du phénomène de l'innovation: une réalité abordée par les économistes institutionnalistes ", La recherche sur l'innovation; une boite de Pandore? Cahiers de l'ACFAS, $n^{\circ} 83$, p. 79-113; Tremblay, D.-G. (1992). «Innovation et marchés internes du travail dans le secteur bancaire : vers un modèle multidimensionnel de l'innovation ", Technologies de l'information et société, vol. 4, $\mathrm{n}^{\circ} 3$, oct. 1992, Paris, Dunod, p. 351-380.

7 Tremblay, D.-G. (2006). Networking, Clusters and Human Capital: A Review of Recent Theoretical Developments, conférence présentée au séminaire organisé par le Canadian Policy Research Network et Statistique Canada sur le capital humain, Ottawa, le 13 janvier 2006. Parue sous forme de Note de recherche du réseau CPRN et sur: www.teluq.uqam.ca/chaireecosavoir.

8 Tremblay, D.-G. (2006), Idem.

9 Emanuel, C. (1989). «Le polymorphisme des entreprises et du territoire, une convergence possible des disciplines ", Revue internationale PME, $\operatorname{vol} 2, \mathrm{n}^{\circ} 3$, p. 221-228.

10 Maillat, D. (1989). « Piccole e media, impese innovazione e sviluppo territoriale », Studi e informazioni, $\mathrm{n}^{\circ}$ 3, p. 8599.

${ }^{11}$ Tremblay, D.-G. (2006), op. cit. 